From this central position on Miount Terminillo it will be an easy matter to have chains of small experimental stations going up to the crest $(2,200 \mathrm{~m}$. high -a modest altitude but the highest in the Appennines except for Mount Gran Sasso) and toward the valley of Rieti (where the N. Strampelli Experimental Station for Wheat Culture is situated).

The Niountain Centre is still in a preliminary phase of organisation, since the building which will constitute the $u b i$ consistam of the Institute with its laboratories and its rooms for guest research workers is just now being restored from war damage and adapted to new functions. The Italian Government has leased the building for six years to the University of Pavia, and the Ministry of Public Works has given the funds for restoring it. The town of Rieti, besides giving up in favour of the Centre every right to the building, which is situated on its own land, has granted several hectares of surrounding ground to be used for experimental fields and other needs of the Centre. The National Council of Research has helped by adding to the annual grant to the Centre of Genetics the sum of 100,000 lire in order to pay the rent and upkeep of the building. A call will be made to Italian industries asking for generous gifts in order to furnish the laboratories and the rooms for the guest research workers, who may be expected to stay at the Centre for some months during the season suitable for their own particular researches, working on plant or animal genetics. The exploration of the flora of Mount Terminillo has been already undertaken, and a herbarium, systematic and topographical schedules, and so on, have been established. A parallel exploration of the fauna, particularly the insects, is planned. The investigation of the flora and fauna is an indispensable preliminary phase, to which biologists specializing in various systematic groups will be asked to contribute. On the basis of such investigations, the animals and plants on which genetical research and the study of evolution can be most fruitfully carried out will be selected.

The scope of the Institute is fundamentally a scientific one in the field of general and theoretical biology ; but without doubt the results of the research work will prove important also for agriculture, and collaboration is already planned with other institutions for such purposes as applications to sylviculture, forage problems, and so on.

The fitting up of the Institute, and even more, its activities and functions, will require substantial financial support. But confidence prevails with regard to the possibility of securing adequate support, since the interest of the undertaking is quite clear, and the Institute might well assume an international character. The importance of the general problems which the Institute proposes to investigate in its experimental activities, the structure of the Institute itself, founded on co-operation between research workers specializing in various groups of plants and animals, the fact that in Europe there is no institute with an organisation corresponding to the system of the three transplant stations that the Carnegie Institution of Washington supports in California (located at Stanford University near the coast, at Mather half-way up the Sierra Nevada and at Timberline near the crest of this mountain range) - these and other conditions make the Mountain Centre of Genetics suitable for consideration as one of the international institutes of research, the establishment of which the Economic and Social Council of the United Nations is prepared to encourage.

\section{SHORT-PERIOD VARIATIONS IN THE IONOSPHERE}

\author{
By G. H. MUNRO
}

Electrical Engineering Department, University of Sydney

$\mathrm{D}^{\mathrm{u}}$ URING the years 1938 and 1939 fixed-frequency pulse obsefvations were being used to record continuously the virtual height and echo intensity of reflexions fyom the $F^{\prime}$-region at two frequencies close to the veptical penetration frequency for the region. It was poticed that, in the daytime, the penetration frequoncy often showed quasi-periodic fluctuations with periods of the order of half an hour. The magnitude of the fluctuation was variable but frequently of the order of $1 \mathrm{Mc}$./s. from maximum to minimum.

In endeavouring to correlate these effects with changes in $h^{\prime} f$ records then being taken at Mount Stromlo, Canberra, Mr. A. J. Higgs, of the Commonwealth Observatory, found that they appeared to be initial indications of jonization changes which showed a progression down the $h^{\prime} f$ curve from the higher to the lower frequencies. At this stage the investigation was interrupted by diversion of staff to warwork.

During the past year an investigation of horizontal movements in the $F$-region was undertaken ${ }^{1}$, and ionization changes were observed which appeared to have a progression in a horizontal direction. It was also observed that these were frequently quasiperiodic in nature with periods of the same order as in those mentioned above.

By taking $h^{\prime} f$ records in conjunction with the three. point observations, we have now been able to estab. lish quite definitely that all these effects are due to local fluctuations in ionization in the $F$-region, which show both a vertical progression downwards and a horizontal progression.

The $h^{\prime} f$ recorder, which was kindly made available for a short period by the Radiophysics Laboratory of the Council for Scientific and Industrial Research, was located at Liverpool (point 4 in my previous communication, ref. 1), and observational runs were taken continuously (repeating every two minutes), simultaneously with observations on the three-point system.

A good set of records was obtained on June 22, 1948. Fig. 1 shows a set of $h^{\prime} f$ tracings illustrating the sequence of events during 1230-1314. It will be seen that kinks appear in the $h^{\prime} f$ curves and move down from the higher to the lower frequencies. These imply corresponding variations in the vertical ionization gradient.

The sequence of events is as follows. At 1230 the ordinary ray curves look to be approximately what would be expected from a regular parabolic gradient of ionization. From 1230 to 1242 the penetration frequency falls steadily and the virtual height in. creases for frequencies near penetration. At 1242 a sharp maximum of virtual height appears a little below the penetration frequency. The visible disturbance is thus an increase of virtual height above the normal value for a particular frequency. The maximum of this disturbance progresses down the $h^{\prime} f$ curve with time, being obvious again at 1314 as the virtual height maximum at $6 \mathrm{Mc} . / \mathrm{s}$.

The curve for the extraordinary ray shows similar changes, but later in time, causing cross-over points of the two rays which also move down the curves. 
No. 4151 May 21, 1949

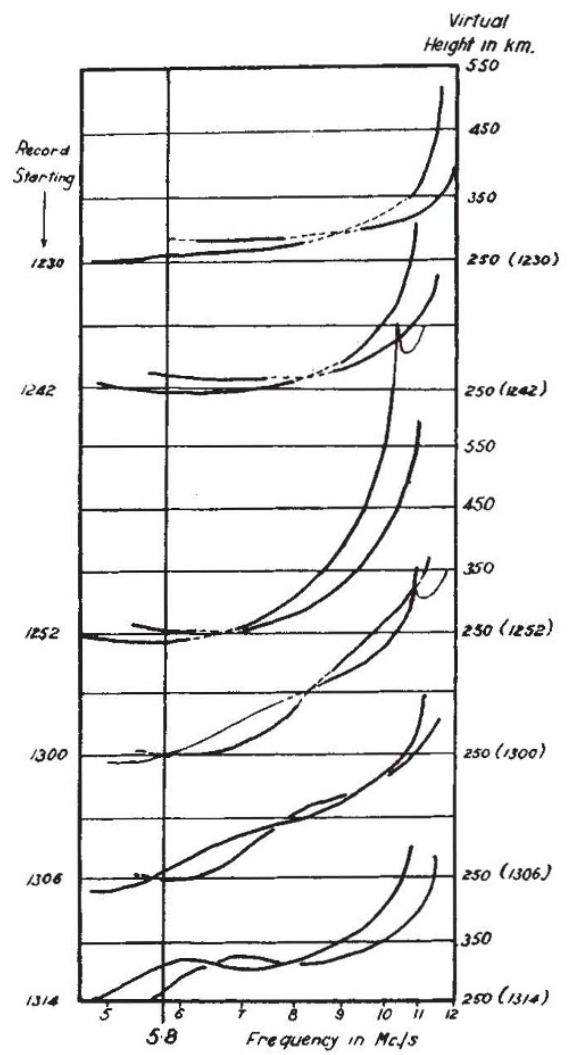

Fig. 1. Downward progression of ionospheric disturbances on $h^{\prime} f$ curves. (Point 4, June 22, 1948)

The record on a fixed frequency of $5.8 \mathrm{Mc} / \mathrm{s}$. will show the changes in virtual heights of the two com. ponents at this frequency, giving a pattern similar to that of Fig. 4 in my previous communication (ref. 1).

To examine the nature of the disturbance more carefully, we have used the method outlined by Manning ${ }^{2}$ to convert the virtual height/frequency curves to true height/frequency curves and so derive the true height/ionization density curves. From these we are able to plot changes in ionization density with time for any actual height. A set of such curves is shown in Fig. 2 for the same period as shown in Fig. 1.

It will be seen that at each height the ionization passes through a maximum and then a minimum, and that these maxima and minima progress downward at a substantially uniform rate of approximately $3.5 \mathrm{~km}$. per minute.

The apparent horizontal velocity of the disturbance at Sydney as determined from the three-point system on $5.8 \mathrm{Mc} . / \mathrm{s}$. was approximately $7 \mathrm{~km}$. per minute.

In order to determine the lateral extent of these effects we have examined the $h^{\prime} f$ records for Canberra for the same day. These show very similar effects to those observed at Sydney, but occurring approximately $15 \mathrm{~min}$. earlier.

The bearing of Sydney from Canberra is $37^{\circ}$ (E. of N.), and the mean direction of apparent horizontal movement observed at Sydney on the three-point system was $28^{\circ}$. The distance is $237 \mathrm{~km}$., so that the apparent horizontal velocity deduced from the Canberra-Sydney time difference is approximately $16 \mathrm{~km}$. per min., compared with $7 \mathrm{~km}$. per min. deduced from the Sydney three-point observations.
We have examined also the Brisbane records for the same day (bearing approximately $10^{\circ}$, and dis. tance approximately $700 \mathrm{~km}$.). Similar effects do appear there, but, owing to the distance, correlation cannot be established as certainly as in the case of Canberra.

Considering the simplest explanations, the effects observed could be due to the progression of a pressure wave with the wave-front having an appropriate tilt with reference to the earth's surface. The observed velocities are of the right order for this. They could also be the result of an atmospheric drift with a varying ionization gradient superimposed on it. Further investigations are in progress to enable better assessment of the probabilities.

The difference in the apparent horizontal velocities as deduced from $(a)$ the Canberra-Sydney timedifference, and $(b)$ the three-point observations at Sydney, could be explained by variations in velocity, direction or tilt over the intervening distance.

Disturbances of this type appear to occur on most days at all sites from which suitable records are available to us. It seems most important, therefore, that examination of apparent velocities should be made, for as many locations as possible, to establish the extent and variations of the effects with the view of establishing the origin of the disturbances.

These disturbances are of direct practical interest in connexion with radio propagation via the ionosphere. The continually varying difference in paths

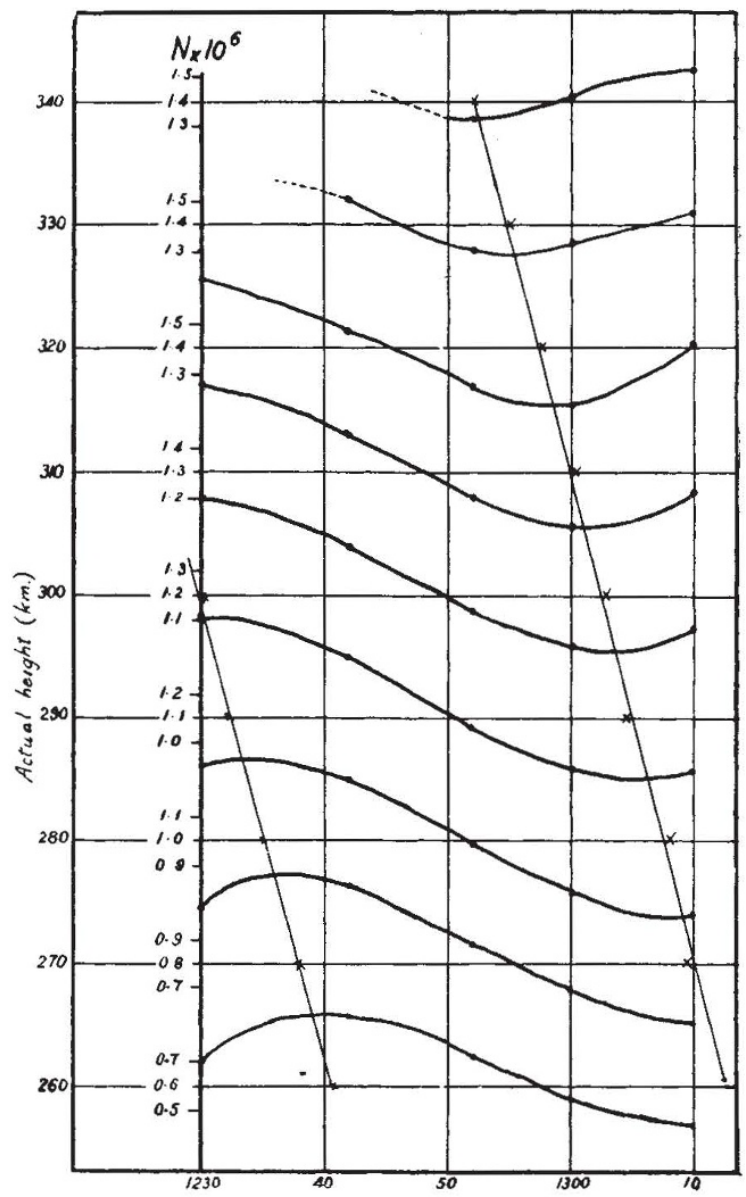

Fig. 2. Changes in ionization at various actual heights. (Point 4, June 22, 1948) 
of the ordinary and extraordinary rays causes interference beats between the two which results in severe fading in receivers. In addition, it is found that the disturbances cause large variations in the strength of the returned signal.

The investigations are continuing, and will be reported more fully elsewhere.

This work has been carried out in the Electrical Engineering Department of the University of Sydney as part of the programme of the Radio Research Board of the Commonwealth Council for Scientific and Industrial Research, and is published with the consent of the Council.

The keen interest of Sir John Madsen, chairman of the Board, and the assistance of the staff of the Sydney Section, are gratefully acknowledged.

${ }^{1}$ Nature, 162, 886 (1948).

2 Proc. Inst. Rad. Eng. (Nov. 1947).

\section{6}

\section{CONSTITUTION OF THE TERRESTRIAL PLANETS}

TN a paper, "On thelfonstitution of the Terrestrial I Planets", by. H. Ramsey (Mon. Not. Roy. Astro. Soc., (10, 5 ; 1948), it is suggested that the

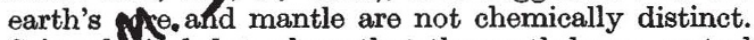
Seismold gical data show that the earth has a central ofe yohich occupies about one-sixth of its total folme and contains about one-third of its mass, and it has been generally assumed that the material of this core is an alloy of iron and nickel. The core is mainly responsible for the earth's high mean density - the highest in the solar system. The high masses of the four great outer planets have enabled them to retain hydrogen and other volatile substances, and this explains their comparatively low densities; but the fact that the terrestrial planets have mean densities smaller than that of the earth presents a difficulty.

One explanation would be that the planets were originally of different compositions ; but this theory is impossible to reconcile with any current theory of the origin of the solar system. The only other explanation is that the earth's core and mantle are not chemically distinct, and Ramsey's analysis supports this view. He assumes that the hydrostatic pressure at the boundary of the earth's core depends only on the chemical and crystallographic properties of the substances concerned, which must be true if the core and mantle of the earth are respectively metallic and molecular phases of the same chemical substance.

Assuming, then, that the pressure at the boundary of the core is the same for all terrestrial planets, the mean densities of the terrestrial planets are computed, and these are in good agreement with the empirical results; the calculated density of the moon is also in excellent agreement with the observed value. The greatest discrepancy occurs in the case of Mars, being about ten per cent; but this and other smaller discrepancies can be explained if allowance is made for the tendency of the heavier elements to gravitate towards the earth's centre. While the theory can account for the mean densities of the planets in a manner which is satisfactory from the point of view of cosmogony, it is admitted that it cannot be regarded as completely established until the pressure characteristic of the boundary of the core has been calculated from atomic theory.

\section{CONSERVATION AND UTILIZA- TION OF WORLD RESOURCES}

$\mathrm{T}$

HE provisional programme of the United Nations Scientific Conference on the Conservation and Utilization of Resources to be held at Lake Success during August 17-Sertember 6, 1949, has now been issued to mef ber Dovernments for comment, and in particular prostions for addition or deletion of tofids. The primary concern of the Conference is withe particular application of science to the man agenent and use of resources, and the knowledge of numerous related sciences will be brought into full play on single problems so as to facilitate complete and not partial solutions. The plenary meetings will discuss techniques for assessing and classifying resources; for protecting resources, whether by reducing waste in mineral resources, by control of injurious insects or diseases, or by prevention of soil erosion; and for increasing production; as well as techniques of special interest to the less developed countries, the special scientific and technical needs of such countries, and experience in the interrelated application of such techniques, for example, as the Tennessee Valley Authority.

A section will hold meetings to consider the inventory and survey of forests, control of fire, insects and diseases in forests, sylvicultural techniques, the organisation of research and education in forestry, forest administration, manufacturing processes for wood, and the utilization of wood-waste and new products from wood. Another section will consider the appraisal of water resources, watershed management, multiple-purpose reservoirs, pollution, salinity and sedimentation of water supplies, methods of controlling floods, the relation of soils to irrigation and drainage, and irrigation and drainage technique generally.

Methods and techniques for the conservation and use of soil, soil productivity and fertilizers will be discussed in a third section, which will also consider research programmes and surveys in this field, the settlement of new lands, new crops and plant breeding as techniques for conservation, cropping systems, protection of crops and grasslands against weeds, diseases and pests, storage and preservation of agricultural products, adaptation of livestock to environment and its improvement through breeding, the feeding of livestock, diseases and pests of livestock, farming systems in relation to soil conservation, the condition of grazing lands, and seeding of natural grazing lands. A fourth section will consider such problems of fisheries and wild life as changes in abundance of marine populations, the utilization and management of marine fishery resources as well as inland fisheries, cultivation of aquatic animals, the place of fish and wildfowl conservation in the balanced development of resources, and the control of injurious animals.

A fifth section, on fuels, will consider techniques of producing coal, oil and gas, the recovery of secondary oil, synthetic fuels, and the utilization of coal resources, including by-products. The sixth section, on power, will consider the various methods of power production, including steam and Diesel-electric plants, the gas turbine, hydro-electric power and the wind turbine, transmission, distribution and utilization problems. The seventh section, on mineral resources, will consider the measurement of such resources, methods of prospecting or discovery, new 